\title{
On certain subclasses of bounded univalent functions
}

\author{
by J. FukA (Praha) and Z. J. JAKubowski (Łódź)
}

\begin{abstract}
Let $\mathbb{D}=\{z \in \mathbb{C} ;|z|<1\}, T=\{z \in \mathbb{C} ;|z|=1\}$. Denote by $S$ the class of functions $f$ of the form

$$
f(z)=z+a_{2} z^{2}+\ldots
$$

holomorphic and univalent in $\mathbb{D}$, and by $S(M), M>1$, the subclass of functions $f$ of the family $S$ such that $|f(z)|<M$ in $\mathbb{D}$. We introduce (and investigate the basic properties of) the class $S(M, m ; \alpha), 0<m \leq M<\infty, 0 \leq \alpha \leq 1$, of bounded functions $f$ of the family $S$ for which there exists an open $\operatorname{arc} I_{\alpha}=I_{\alpha}(f) \subset T$ of length $2 \pi \alpha$ such that $\varlimsup_{z \rightarrow z_{0}, z \in \mathbb{D}}|f(z)| \leq M$ for every $z_{0} \in I_{\alpha}$ and $\varlimsup_{z \rightarrow z_{0}, z \in \mathbb{D}}|f(z)| \leq m$ for every $z_{0} \in T \backslash \bar{I}_{\alpha}$.
\end{abstract}

1. Let $\mathbb{D}=\{z \in \mathbb{C} ;|z|<1\}, T=\{z \in \mathbb{C} ;|z|=1\}$. Denote by $S$ the class of functions $f$ holomorphic and univalent in $\mathbb{D}$ of the form

$$
f(z)=z+a_{2} z^{2}+\ldots+a_{n} z^{n}+\ldots,
$$

and by $S(M), M>1$, the subclass of functions $f$ of the family $S$ such that $|f(z)|<M$ in $\mathbb{D}$.

Usually subclasses of $S$ are defined by natural geometric conditions, for example the subclass $S^{c}$ of convex functions or $S^{*}$ of starlike functions, and then these conditions are formulated analytically in all of $\mathbb{D}$. In 1969 P. Mocanu ([3]) introduced a new class $M_{\alpha}, 0 \leq \alpha \leq 1$, of functions of the form (1.1) by joining "homotopically" the classes $S^{c}$ and $S^{*}$. Analogous constructions were used by other authors for other couples of classes examined in geometric function theory (see for instance [5]). The essence of such considerations consists in the following: the new class is defined by an analytic condition which holds at every point of $\mathbb{D}$ and for given values of the parameter reduces to the analytic conditions defining the classes being joined. For instance, in the paper of Mocanu [3] we have $M_{0}=S^{*}, M_{1}=S^{c}$

1991 Mathematics Subject Classification: Primary 30C55. 
and the joining condition reads

$$
\operatorname{Re}\left\{(1-\alpha) z f^{\prime}(z) / f(z)+\alpha\left(1+z f^{\prime \prime}(z) / f^{\prime}(z)\right)\right\}>0, \quad z \in \mathbb{D} .
$$

In the course of discussions another idea how to join a couple of subclasses of $S$ crystallized: the analytical conditions have to be fulfilled on some arcs of $T$. Let us sketch this general idea. Let $S_{0}\left(S_{1}\right)$ be the class of all functions of the form (1.1) fulfilling on the circle $T$ a certain condition $W_{0}$ $\left(W_{1}\right)$. The class $S_{\alpha}, \alpha \in(0,1)$, of functions of the form (1.1) is then defined in the following manner: for every $f \in S_{\alpha}$ there exists an $\operatorname{arc} I_{\alpha}(f) \subset T$ of length $2 \pi \alpha$ such that $f$ fulfils $W_{1}$ on $I_{\alpha}(f)$ and $W_{0}$ on the rest of $T$. As $\alpha \rightarrow 0^{+}, S_{\alpha}$ evidently "tends" to $S_{0}$, and as $\alpha \rightarrow 1^{-}$to $S_{1}$.

In this paper we try to implement the above idea. Although the results are simple and incomplete, we have decided to publish them in the hope that the idea could attract the attention of the reader or stimulate his own reflections.

2. Definition. Let $0<m \leq M<\infty, 0 \leq \alpha \leq 1$ and let $f \in S$ be bounded. We say that $f \in S(M, m ; \alpha)$ if there exists an open $\operatorname{arc} I_{\alpha} \subset T$ of length $2 \pi \alpha$ such that for every $z_{0} \in I_{\alpha}$

$$
\varlimsup_{z \rightarrow z_{0}, z \in \mathbb{D}}|f(z)| \leq M
$$

and for every $z_{0} \in T \backslash \bar{I}_{\alpha}$

$$
\varlimsup_{z \rightarrow z_{0}, z \in \mathbb{D}}|f(t)| \leq m .
$$

The following properties are obvious:

$$
\begin{array}{ll}
S(M, m ; \alpha)=S(m) & \text { for } \alpha=0, \\
S(M, m ; \alpha)=S(M) & \text { for } \alpha=1, \\
S(M, M ; \alpha)=S(M) & \text { for every } \alpha \in[0,1], \\
S\left(M_{1}, m ; \alpha\right) \supset S\left(M_{2}, m ; \alpha\right) & \text { if } M_{1} \geq M_{2}, \\
S\left(M, m_{1} ; \alpha\right) \supset S\left(M, m_{2} ; \alpha\right) & \text { if } m_{1} \geq m_{2} .
\end{array}
$$

If $f \in S(M, m ; \alpha)$, then $f$ is bounded and fulfils (2.1) at every point $z_{0} \in T$ except the endpoints of $I_{\alpha}$. So by the generalized maximum principle $|f(z)| \leq M$ in $\mathbb{D}$, hence $f \in S(M)$. Consequently,

$$
S(M, m ; \alpha) \subset S(M) \quad \text { for every } \alpha \in[0,1] .
$$

From (2.1)-(2.4') it follows directly that for $0 \leq \alpha_{1} \leq \alpha_{2} \leq 1$

$$
S(m) \subset S\left(M, m ; \alpha_{1}\right) \subset S\left(M, m ; \alpha_{2}\right) \subset S(M) .
$$

3. The essential problem is to characterize the conditions on the parameters $M, m, \alpha$ under which the classes $S(M, m ; \alpha)$ are not void or trivial. 
From $\left(2.4^{\prime}\right)$ it follows that $M>1$, and from $\left(2.4^{\prime \prime}\right)$ that $S(M, m ; \alpha) \neq \emptyset$ if $m \geq 1$.

Also (for $\alpha<1$ ) the inequality

$$
m \geq a(M)=M\left(2 M-1-2 \sqrt{M^{2}-M}\right)
$$

has to be valid. This follows from $(2.2),\left(2.4^{\prime}\right)$ and from the covering theorem of G. Pick in the class $S(M)([4])$.

It is possible to obtain a general necessary condition on the parameters $M, m, \alpha$ for $S(M, m ; \alpha) \neq \emptyset([1])$.

Theorem 1. If $S(M, m ; \alpha) \neq \emptyset$, then

$$
M^{\alpha} m^{1-\alpha} \geq 1 .
$$

Proof. Let $f \in S(M, m ; \alpha)$. Because $f$ is of the form (1.1), holomorphic and bounded in $\mathbb{D}$, the function $\phi(z)=f(z) / z, 0 \neq z \in \mathbb{D}, \phi(0)=f^{\prime}(0)=1$, is also holomorphic and bounded in $\mathbb{D}$. But from (2.1) and (2.2) it follows that $\phi$ also fulfils (2.1) and (2.2). Hence the theorem on two constants ([2], Chapter 6, p. 204) gives

$$
|\phi(z)| \leq M^{\omega(z)} m^{1-\omega(z)}, \quad z \in \mathbb{D},
$$

where $\omega(z)$ is the harmonic measure of the $\operatorname{arc} I_{\alpha}$ at the point $z$. Since $\phi(0)=1$ and $\omega(0)=\alpha,(3.2)$ follows from (3.3).

From (3.1) and (3.2) one obtains the following possible ranges for the parameters $M, m, \alpha$ :

$$
1<M<\infty, \quad a(M) \leq m<1, \quad 0<\frac{\log (1 / m)}{\log (M / m)} \leq \alpha \leq 1,
$$

or

$$
1 \leq M<\infty, \quad 1 \leq m \leq M, 0 \leq \alpha \leq 1 .
$$

As was mentioned above, in the case (3.5) we always have $S(M, m ; \alpha) \neq \emptyset$ (see $\left(2.4^{\prime \prime}\right)$ ). If $1 \leq m \leq M$, the inequality (3.2) is fulfilled for every $\alpha \in[0,1]$ and therefore it is uninteresting.

4. Let

$$
k(z)=z /(1-z)^{2}, \quad z \in \mathbb{D},
$$

be the Koebe function and

$$
p(z ; M)=M k^{-1}[k(z) / M], \quad z \in \mathbb{D}, M \geq 1,
$$

be the Pick function. Evidently $p(z ; 1)=z$ and $p(z ; M) \in S(M)$. It is well known that $p$ conformally maps $\mathbb{D}$ onto the $\operatorname{disc}\{w \in \mathbb{C} ;|w|<M\}$ with a slit along the segment $(-M,-a(M)]$. 
Take $t \in[1, M], M>1$, and let $M_{t}$ be defined by $k\left(M_{t}\right)=t /(4 M)$, i.e. from (4.1)

$$
M_{t}=-\frac{\sqrt{M}-\sqrt{M-t}}{\sqrt{M}+\sqrt{M-t}} \quad\left(M M_{M}=-M, M M_{1}=-a(M)\right) .
$$

Then $M_{t}$ is a decreasing function of $t$. Set

$$
z_{t}=1-\frac{2}{t}+\frac{2 i \sqrt{t-1}}{t} .
$$

It is easy to observe that $z_{t} \in T$. Further, denote by $\left(z_{t},-1, \bar{z}_{t}\right) \subset T$, $t \in(1, M]$, the closed arc containing -1 with endpoints $z_{t}, \bar{z}_{t}$. The function (4.2) maps the arc $\left(z_{t},-1, \bar{z}_{t}\right)$ on the twice covered segment $\left[M M_{t}\right.$, $\left.M M_{1}\right]$ and $p\left(z_{t} ; M\right)=p\left(\bar{z}_{t} ; M\right)=M M_{t}, p(-1 ; M)=M M_{1}=-a(M)$.

Denote by $2 \pi(1-\alpha)$ the length of the arc $\left(z_{t},-1, \bar{z}_{t}\right)$. Then $2 \pi(1-\alpha)=$ $2\left(\pi-\arg z_{t}\right)$, i.e.

$$
\alpha=(1 / \pi) \arg z_{t}
$$

From (4.4) we obtain

$$
\arg z_{t}= \begin{cases}\pi-\arctan b(t) & \text { for } 1<t<2 \\ \pi / 2 & \text { for } t=2 \\ -\arctan b(t) & \text { for } 2<t\end{cases}
$$

where

$$
b(t)=\frac{2 \sqrt{t-1}}{2-t}, \quad t>1, t \neq 2
$$

Moreover,

$$
0<\arg z_{M} \leq \arg z_{t}<\pi \quad \text { for } 1<t \leq M .
$$

From (4.5) and (4.8) we have

$$
(1 / \pi) \arg z_{M} \leq \alpha<1 \quad \text { for } 1 \leq t \leq M .
$$

Consider three cases:

I. $M \in(1,2)$. Then for every $t \in(1, M]$ from (4.5), (4.6) we obtain

$$
\alpha=1-(1 / \pi) \arctan b(t)
$$

and

$$
\alpha \in[1-(1 / \pi) \arctan b(M), 1) .
$$

Conversely, if $\alpha$ fulfils (4.11), then the equation (4.10) has in $(1, M]$ the unique solution

$$
t=2(1+\cos \pi \alpha) / \sin ^{2} \pi \alpha .
$$


II. $M=2$. Then for every $t \in(1,2]$ we have

$$
\alpha= \begin{cases}1-(1 / \pi) \arctan b(t) & \text { for } t \in(1,2), \\ 1 / 2 & \text { for } t=2,\end{cases}
$$

and $\alpha \in[1 / 2,1)$. Conversely, from (4.13) we obtain

$$
t= \begin{cases}2(1+\cos \pi \alpha) / \sin ^{2} \pi \alpha & \text { for } \alpha \in(1 / 2,1), \\ 2 & \text { for } \alpha=1 / 2 .\end{cases}
$$

III. $M>2$. Then for every $t \in(1, M]$ we have

$$
\alpha= \begin{cases}1-(1 / \pi) \arctan b(t) & \text { for } t \in(1,2), \\ 1 / 2 & \text { for } t=2, \\ -(1 / \pi) \arctan b(t) & \text { for } t \in(2, M),\end{cases}
$$

and

$$
\alpha \in[-(1 / \pi) \arctan b(M), 1) .
$$

Conversely, if $\alpha$ fulfils (4.16), then $t$ is given by (4.14) and

(4.17) $t=2(1+\cos \pi \alpha) / \sin ^{2} \pi \alpha \quad$ for $\alpha \in[-(1 / \pi) \arctan b(M), 1 / 2)$.

From the above considerations it follows that for every $z \in\left(z_{t},-1, \bar{z}_{t}\right)$

$$
|p(z ; M)| \leq M\left|M_{t}\right|=M \frac{\sqrt{M}-\sqrt{M-t}}{\sqrt{M}+\sqrt{M-t}} .
$$

Let $M \in(1, \infty), m \in(a(M), M]$. From (4.3) it follows that there exists exactly one $t=t_{m} \in(1, M]$ such that

$$
m=M \frac{\sqrt{M}-\sqrt{M-t}}{\sqrt{M}+\sqrt{M-t}} .
$$

Consequently, from (4.18) and (4.19) we obtain $|p(z ; M)| \leq m$ on the $\operatorname{arc}\left(z_{t_{m}},-1, \bar{z}_{t_{m}}\right)$. Taking $t=t_{m}$ in (4.10), (4.13), (4.15), respectively, we obtain $\alpha_{m}$. Hence we have proved

TheOREM 2. Let $M \in(1, \infty)$ and suppose $m \in(a(M), M]$. Let $t=$ $t(M, m) \in(1, M]$ be the solution of the equation (4.19) and let $\alpha=\alpha_{t}$ be given by (4.15), where the functions $a(M), b(t)$ are defined by (3.1), (4.7), respectively. Then the Pick function (4.2) belongs to the class $S\left(M, m, \alpha_{t}\right)$.

From the inclusion $\left(2.4^{\prime \prime}\right)$ at once follows

Corollary 1. If $\alpha \in\left[\alpha_{t}, 1\right]$, then $p(z ; M) \in S(M, m ; \alpha)$, where $\alpha_{t}$ is defined in Theorem 2.

Remark. The above considerations can be reversed. Let $M>1$ and suppose $\alpha$ lies in the interval (4.9) (compare (4.6)). Then take $t$ from (4.12), (4.14), (4.17), respectively, and $m$ from (4.19). Then clearly $p(z ; M) \in S(M, m ; \alpha)$. From $(2.3)$ we also have $p(z ; M) \in S(M, \widetilde{m} ; \alpha)$ for 
every $\widetilde{m} \geq m$. Evidently the inequality (4.9) imposes here an essential restriction (compare (3.4)). Hence there remains the question whether the classes $S(M, m ; \alpha)$ are nonvoid for all remaining values of the parameters $M, m, \alpha$ fulfilling the conditions (3.4) and not covered by Theorem 2 .

5. Denote by $\mathbb{A}$ the set of all 3 -tuples $(M, m, \alpha)$ such that $p(z ; M) \in$ $S(M, m ; \alpha)$. From $\left(2.4^{\prime \prime}\right)$ we have $S(M, m ; \alpha) \subset S(M)$. Hence if the Pick function maximizes a given functional $H(f)$ on the class $S(M)$, then evidently

$$
\max _{f \in S(M, m ; \alpha)} H(f)=H(p)
$$

for $(M, m, \alpha) \in \mathbb{A}$. In particular, from the well known results (see for instance [4]) we obtain

Corollary 2. If $f \in S(M, m ; \alpha)$ and $(M, m, \alpha) \in \mathbb{A}$, then

$$
\left|a_{2}\right| \leq\left|a_{2 p}\right|=2\left(1-M^{-1}\right) \text {. }
$$

Corollary 3. If $f \in S(M, m ; \alpha),(M, m, \alpha) \in \mathbb{A},|z|=r, r \in(0,1)$ and $k(z)$ is the Koebe function (4.1), then

$$
q_{M} \leq|f(z)| \leq \rho_{M},
$$

where $\rho_{M} \in(0, r M), q_{M} \in(0, M)$ and

$$
k\left(\rho_{M} / M\right)=k(r) / M, \quad k\left(-q_{M} / M\right)=k(-r) / M .
$$

TheOREM 3. The class $S(M, m ; \alpha)$ is compact in the topology of uniform convergence on compact subsets of $\mathbb{D}$.

Proof. 1) Let $\left\{f_{n}\right\}_{n=1}^{\infty}$ be a sequence in $S(M, m ; \alpha)$ with the following properties:

(i) $\varlimsup_{z \rightarrow z_{0}, z \in \mathbb{D}}\left|f_{n}(z)\right| \leq M$ for every $z_{0}$ in the open arc $I_{n}=I_{\alpha}\left(f_{n}\right) \subset T$ and $\varlimsup_{z \rightarrow z_{0}, z \in \mathbb{D}}\left|f_{n}(z)\right| \leq m$ for every $z_{0}$ in the complementary arc $T \backslash \bar{I}_{n}$.

(ii) $I_{n} \rightarrow I$ in the following sense: if $x_{n}, y_{n}$ and $x, y$ are the endpoints of $I_{n}$ and $I$, respectively, then $x_{n} \rightarrow x, y_{n} \rightarrow y$ and, for every $\zeta \in I$, there exists $n_{\zeta}$ such that $\zeta \in I_{n}$ for $n \geq n_{\zeta}$. Similarly, $T \backslash \bar{I}_{n} \rightarrow T \backslash \bar{I}$.

(iii) $f_{n} \rightarrow f$ uniformly on compact subsets of $\mathbb{D}$.

We shall show that $f \in S(M, m ; \alpha)$. By the theorem on two constants, we have, for every $z \in \mathbb{D},\left|f_{n}(z)\right| \leq M^{\omega_{n}(z)} m^{1-\omega_{n}(z)}$ where $\omega_{n}(z)$ is the harmonic measure of the arc $I_{n}$. Of course,

$$
\omega_{n}\left(r e^{i \theta}\right)=\frac{1}{2 \pi} \int_{-\pi}^{\pi} P_{r}(\theta-t) \chi_{I_{n}}(t) d t
$$

where $\chi_{I_{n}}$ is the characteristic function of $I_{n}$ and $P_{r}(\phi)$ is the Poisson kernel. From (ii) it follows that $\chi_{I_{n}}(\xi) \rightarrow \chi_{I}(\xi)$ for every $\xi \in T \backslash\{x, y\}$. Hence from 
the Lebesgue dominated convergence theorem we have, for each $z \in \mathbb{D}$,

$$
\omega_{n}(z) \rightarrow \frac{1}{2 \pi} \int_{-\pi}^{\pi} P_{r}(\theta-t) \chi_{I}(t) d t=\omega(z) \quad \text { as } n \rightarrow \infty .
$$

So, for every $z \in \mathbb{D}$, we have from (iii)

$$
|f(z)|=\lim _{n \rightarrow \infty}\left|f_{n}(z)\right| \leq \lim _{n \rightarrow \infty} M^{\omega_{n}(z)} m^{1-\omega_{n}(z)}=M^{\omega(z)} m^{1-\omega(z)} .
$$

From the continuity of $\omega(z)$ on $I$ and on $T \backslash \bar{I}$, we finally obtain

$$
\varlimsup_{z \rightarrow \zeta, z \in \mathbb{D}}|f(z)| \leq \lim _{z \rightarrow \zeta, z \in \mathbb{D}} M^{\omega(z)} m^{1-\omega(z)}=M \quad \text { for } \zeta \in I,
$$

and similarly,

$$
\varlimsup_{z \rightarrow \zeta, z \in \mathbb{D}}|f(z)| \leq m \quad \text { for } \zeta \in T \backslash \bar{I}
$$

(cf. (2.1) and (2.2)). Since from (iii) $f^{\prime}(0)=1$, we conclude, by the theorem of Hurwitz, that $f$ is univalent in $\mathbb{D}$, and so $f \in S(M, m ; \alpha)$.

2) Let $\left\{f_{n}\right\}_{n=1}^{\infty}$ be an arbitrary sequence of functions in $S(M, m ; \alpha)$. Choosing a subsequence $\left\{f_{n_{k}}\right\}$ converging uniformly on compact subsets of $\mathbb{D}$ to a holomorphic function $f$ (cf. $\left(2.4^{\prime}\right)$ ) and then from $\left\{n_{k}\right\}$ a subsequence $\left\{m_{l}\right\}$ (by the compactness of $T$ ) so that $I_{m_{l}} \rightarrow I$, we obtain the situation of part 1) of the proof. Theorem 3 is proved.

\section{References}

[1] J. Fuka and Z. Jakubowski, On certain subclasses of bounded univalent functions, in: Proc. of the XI-th Instructional Conference on the Theory of Extremal Problems, Łódź, 1990, 20-27 (in Polish).

[2] A. I. Markushevich, Theory of Analytic Functions, Vol. 2, Nauka, Moscow 1968 (in Russian).

[3] P. T. Mocanu, Une propriété de convexité généralisée dans la théorie de la représentation conforme, Mathematica (Cluj) 11 (1969), 127-133.

[4] G. Pick, Über die konforme Abbildung eines Kreises auf ein schlichtes und zugleich beschränktes Gebiet, Sitzungsber. Akad. Wiss. Wien 126 (1917), 247-263.

[5] K. Skalska, Certain subclasses of the class of typically real functions, Ann. Polon. Math. 38 (1980), 141-152.

MATEMATICKÝ ÚSTAV ČSAV

ŽITNÁ 25

11567 PRAHA 1

CZECHOSLOVAKIA
INSTITUTE OF MATHEMATICS ŁÓDŹ UNIVERSITY BANACHA 22 90-238 ŁÓDŹ, POLAND 